\title{
Primary Orbital Chondromyxoid Fibroma: A Cause of Monosymptomatic Periocular Pain
}

\author{
Louise Hildestad $^{a}$ Steffen Heegaard ${ }^{a}$ b Peter Bjerre Toft ${ }^{a}$ \\ aDepartment of Ophthalmology, Rigshospitalet, University of Copenhagen, Copenhagen, \\ Denmark; 'bepartment of Pathology, Rigshospitalet, University of Copenhagen, \\ Copenhagen, Denmark
}

\section{Keywords}

Monosymptomatic periocular pain · Tumour · Orbit · Chondromyxoid fibroma

\begin{abstract}
Chondromyxoid fibroma (CMF) is a very rare entity, accounting for $<1 \%$ of all bone tumours. So far, only 4 cases have been reported with the orbit as primary location. Here, we present a case of orbital CMF with periocular pain as the presenting symptom and as the only symptom when the tumour recurred after surgery. A 41-year-old man underwent a CT scan and later an MRI as part of evaluation for left periocular pain that had persisted for a year. Clinical examination was normal. CT and MRI revealed an irregular mass in the lateral part of the left orbital roof. The tumour was removed by curettage, and histopathological examination showed a CMF. The pain was absent for 3 months. A second MRI 5 months postoperatively revealed a recurrence, which was removed surgically, resulting in pain alleviation. In conclusion, CMF is a potential cause of periocular pain, and persistent periocular pain with normal clinical findings should warrant a CT or MRI scan.
\end{abstract}

(c) 2021 The Author(s).

Published by S. Karger AG, Basel

\section{Introduction}

Chondromyxoid fibroma (CMF) is a rare benign tumour most commonly found in long bones. It occurs slightly more often in men and has the highest incidence in the second and third decades of life [1]. CMF rarely occurs in the orbit. We have found 4 cases of CMF with origin in the orbital bones in the literature [2-5]. In 3 of these cases, patients presented with proptosis $[2,3,5]$. In 1 patient, headache was the only symptom and there were no positive 
Hildestad et al.: Chondromyxoid Fibroma Causing Periocular Pain

findings on the eye examination [4]. Here, we present a case of orbital CMF with monosymptomatic left periocular pain, which is only the second case described with no abnormal findings on eye examination. The pain disappeared after primary surgery but returned when the tumour recurred 3 months after.

\section{Case Presentation}

A 41-year-old man was examined at the rheumatology department because of joint pain in the back and the left arm. Previous medical history included high blood pressure. An MRI of the spine revealed several spinal disc herniations as the cause of his back pain. Additionally, intermittent pain in the left temple had been present for about a year and was focused around the left eye. There was a slightly reduced sensibility on the lateral side of the left orbit. A CT and MRI of the brain and orbits revealed a partly intraosseous mass in the frontal part of the lateral wall of the left orbit, above the frontozygomatical suture. The mass was $15 \mathrm{~mm}$ in diameter at its largest, was irregular, and showed enhancement after injection of Gadolinium in the form of Gadovist ${ }^{\circledR}$ (shown in Fig. 1).

The patient was referred to the eye department, where examination revealed a visual acuity of 20/20 in both eyes, normal visual fields and normal pupils equally reactive to light. The intraocular eye pressure was normal, there was no proptosis on exophthalmometry and motility was free with no diplopia in any gaze directions. Palpation of the orbital rim was normal, and there was equal retrobulbar resistance. In summary, the only positive finding was slightly dry eyes with a tear film breakup time of $8 \mathrm{~s}$ in both eyes.

It was decided to remove the mass, which was found macroscopically to be a soft, grey tumour that had created a small cavity in the bone. It was removed by curettage.

Histopathological examination demonstrated a highly cellular tumour consisting of spindle and stellate small cells (chondroblasts) with abundant pink cytoplasm lying in a myxoid and chondroid stroma. There were no mitosis, necrosis, or calcifications. No osteoclast-like giant multinucleated cells were encountered at the periphery. On immunohistochemistry, the tumour cells stained positive for actin, S-100, D2-40, and calponin but negative for desmin. The diagnosis of CMF was based largely on the morphological appearance (shown in Fig. 1).

Four weeks after the surgery, the pain had disappeared, but it returned 3 months after the surgery. Another MRI was performed showing scar tissue and a small recurrence of the tumour. The recurrence was removed in a second procedure almost 6 months after the first. During this procedure the recurrence was first removed by curettage and the cavity was then levelled out with a surgical burr. After the intervention, the pain disappeared and it had not reoccurred at the latest follow-up 3 and a half months after the second surgery. Histopathological examination of the second biopsy differed only from the first biopsy in that it was negative for D2-40 on immunohistochemistry and thus confirmed it was a recurrence of the first tumour.

\section{Discussion}

Periocular pain without any clinical findings is a common situation in the ophthalmological clinic. This case illustrates the usefulness of orbital imaging in such cases.

The radiological examinations in this case included both a CT and MRI scan. These 2 types of imaging contribute in different ways in depicting the tumour. On the CT imaging, a boneeroding lesion is present, but to distinguish the borders of the tumour is difficult. On the MRI, the tumour stands out clearly, but the involvement of bone in the expansion is more difficult

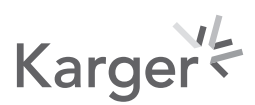




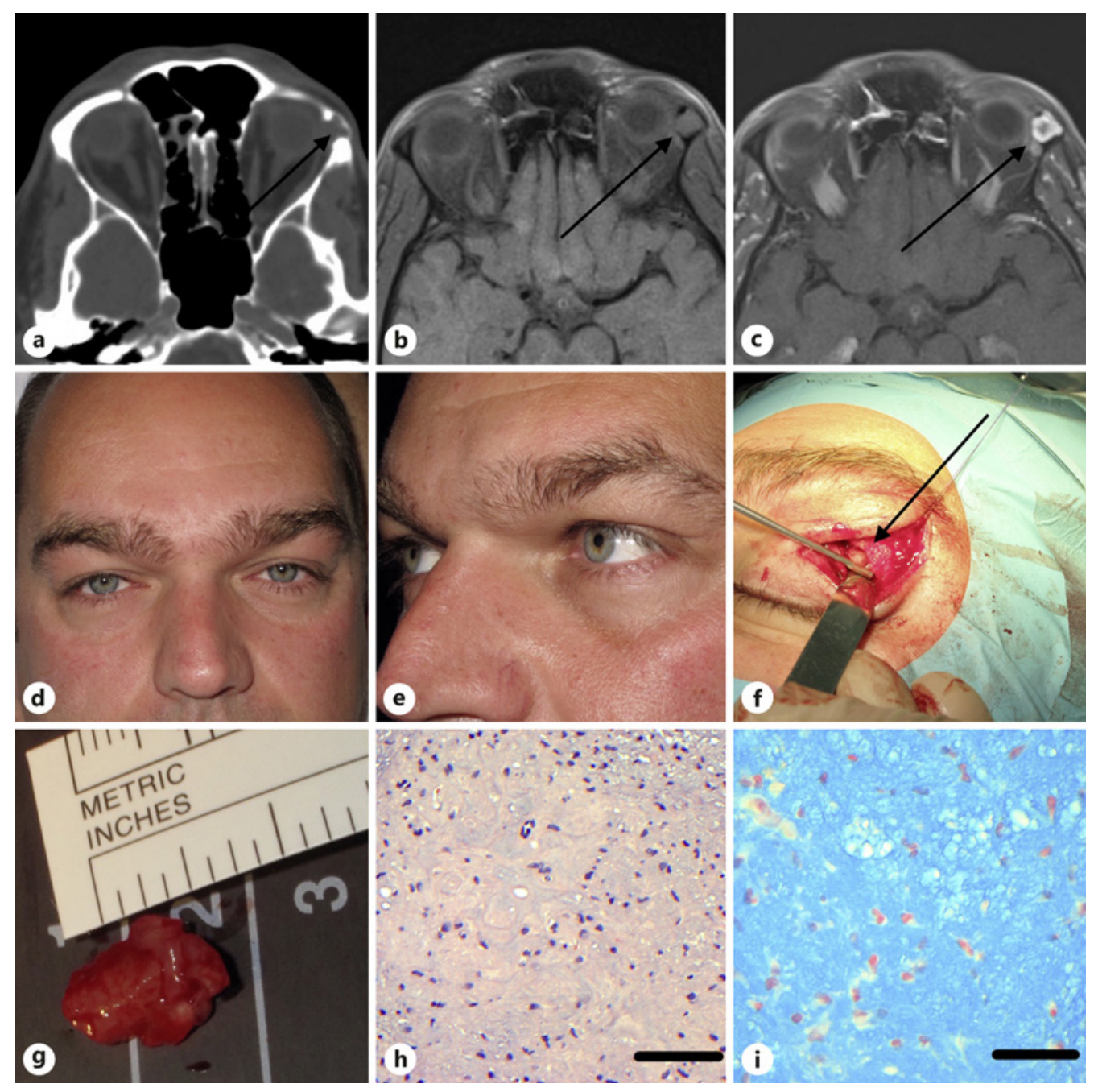

Fig. 1. a A CT scan showed an osteolytic process. On MRI without (b) and with (c) contrast, the tumour was hypointense and showed contrast enhancement. On clinical appearance, the tumour was not visible or palpable, neither before (d) nor after surgery (e). Intraoperative appearance of the tumour located inside the orbital rim (f), and appearance of the non-encapsulated and friable CMF (g). Histology showed spindle cells (chondroblasts) in a myxoid stroma (HE; bar $=100 \mu \mathrm{m})(\mathbf{h})$ and in alcian blue, the myxoid stroma stains blue, while the nuclei are red (alcian blue; bar $=100 \mu \mathrm{m}$ ) (i). CMF, chondromyxoid fibroma.

to make out. Indeed, when seen in the craniofacial sites, CMF often show bone erosion or destruction on imaging, mainly due to the limited room for expansion [6].

CMF is estimated to account for $<1 \%$ of all bone tumours and is rare in the craniofacial sites. In general, bone tumours are rare in the orbit, and covers $<2 \%$ of all orbital tumours [7]. The treatment of CMF is en bloc resection or curettage. The benefits of removal are obtaining the histopathological diagnosis and alleviation of pain.

Previous cases with secondary involvement of the orbit have been described [6, 8]. As mentioned in the introduction, only 4 previous publications describe CMF with origin in the orbit, of which one had no other symptoms than headache. The reason for the perceived pain in these patients is unknown; perhaps it is related to bone erosion. 
In the case described by Ditta et al. [3], a recurrence occurred after 5 months. As in the present case, the tumour had been removed by curettage. Most authors recommend curettage in the skull base and facial bones, as it reduces the need for reconstruction after extensive removal of bone and the cosmetic result is therefore better. The downside is that curettage seems to increase the rate of recurrence by as much as $25 \%$, and because of this surgical treatment should be planned with regards to the possibility of recurrence $[8,9]$.

The necessity of CT or MRI for patients with monosymptomatic periocular pain as in this case could be debated. The prevalence of a finding that explains the headache in cases of a monosymptomatic headache is only between 1.8 and 6.2\% [10]. Based on these numbers, it seems justifiable not to perform CT or MRI initially. However, as illustrated here, cerebral and orbital imaging is worth considering based on the possibility of an orbital tumour also in patients with a normal eye examination.

In summary, CMF is a rare entity in the orbital bones. It can be regarded as a rare differential diagnosis in monosymptomatic periocular pain and illustrates that imaging is worth performing.

\section{Statement of Ethics}

The subject discussed in this paper has given his written informed consent to publish his case, including publication of images. The case complies with the World Medical Association Declaration of Helsinki.

\section{Conflict of Interest Statement}

The authors have no conflicts of interest to declare.

\section{Funding Sources}

This work has not been funded. All authors are employed by Rigshospitalet, University Hospital of Copenhagen.

\section{Author Contributions}

L. Hildestad wrote and edited the paper. S. Heegaard made the histiopathological diagnosis, provided images of the microscopic view of the tumour, and edited the paper. P.B. Toft examined and operated the patient and edited the paper. All authors approve of the final version of this paper.

\section{References}

1 Wu CT, Inwards CY, O'Laughlin S, Rock MG, Beabout JW, Unni KK. Chondromyxoid fibroma of bone: a clinicopathologic review of 278 cases. Hum Pathol. 1998;29(5):438-46.

2 Heindl LM, Amann KU, Hartmann A, Kruse FE, Holbach LM. Orbital chondromyxoid fibroma. Arch Ophthalmol. 2009;127(8):1072-4.

3 Ditta LC, Qayyum S, O’Brien TF, Choudhri AF, Wilson MW. Chondromyxoid fibroma of the orbit. Ophthal Plast Reconstr Surg. 2012;28(5):e105-6.

\section{Karger'₹}


4 Mullen MG, Somogyi M, Maxwell SP, Prabhu V, Yoo DK. Primary orbital chondromyxoid fibroma: a rare case. Ophthal Plast Reconstr Surg. 2017;33(3S Suppl 1):S114-6.

5 Grewal AM, Singh M. Primary chondromyxoid fibroma of the orbit: an orbital mass with calcification. Indian J Ophthalmol. 2019;67(12):2110-3.

6 Meredith DM, Fletcher CDM, Jo VY. Chondromyxoid fibroma arising in craniofacial sites: a clinicopathologic analysis of 25 cases. Am J Surg Pathol. 2018;42(3):392-400.

7 Selva D, White VA, O'Connell JX, Rootman J. Primary bone tumors of the orbit. Surv Ophthalmol. 2004;49(3): 328-42.

8 Khalatbari MR, Hamidi M, Moharamzad Y. Chondromyxoid fibroma of the anterior skull base invading the orbit in a pediatric patient: case report and review of the literature. Neuropediatrics. 2012;43(3):140-5.

9 Castle JT, Kernig ML. Chondromyxoid fibroma of the ethmoid sinus. Head Neck Pathol. 2011;5(3):261-4.

10 Gupta V, Khandelwal N, Prabhakar A, Satish Kumar A, Ahuja CK, Singh P. Prevalence of normal head CT and positive CT findings in a large cohort of patients with chronic headaches. Neuroradiol J. 2015;28(4):421-5. 